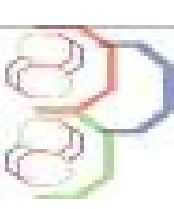

\title{
Variabilité Génétique des Clones de Canne à Sucre Présélectionnées au Stade Ligne en Nouvelle Plantation à Ferké (Nord Côte d'Ivoire)
}

\author{
YYM Béhou*12, CB Péné1, A Zézé2 \\ 1 Direction Recherche et Développement, SUCAF-CI/SOMDIAA, 22, rue des Carrossiers Treichville Zone 3, 01 BP1967 \\ Abidjan 01, Côte d'Ivoire (mbehou@sucafci.somdiaa.com; bpene@sucafci.somdiaa.com ) \\ 2UMRI Sciences Agronomiques et Génie Rural, EDP/INPHB, BP 1093 Yamoussoukro, Côte d'Ivoire (edp@inphb.edu.ci).
}

Original submitted in on 19th April 2018. Published online at www.m.elewa.org on $31^{\text {st }}$ July 2018 https://dx.doi.org/10.4314/jab.v127i1.8

\section{RÉSUMÉ}

Objectifs : L'étude visait à caractériser la diversité génétique en nouvelle plantation d'un lot de 139 variétés de canne à sucre présélectionnées au stade « une ligne ».

Méthodes et résultats : Elle a été conduite en station expérimentale, sous irrigation par aspersion en couverture intégrale, au complexe sucrier de Ferké 1, au Nord Côte d'Ivoire. Ces clones ont été présélectionnées en canne vierge parmi 863 clones plantés par famille, à raison d'un individu par ligne de $3 \mathrm{~m}$ de long sans répétition et comparés à une variété témoin (SP70-1006). Les variables quantitatives et qualitatives relatives à ces clones ont fait l'objet d'une série d'analyses multivariées. L'étude a montré que les variables quantitatives permettant de mieux expliquer la diversité des clones présélectionnées au stade ligne de $3 \mathrm{~m}$ comprenaient, dans l'ordre décroissant, le poids moyen d'une tige, le diamètre moyen des tiges, la longueur moyenne des tiges, le nombre de tiges usinables/3m et le brix. Ces variables ont fortement contribué à la discrimination de 10 groupes phénotypiques de clones. Les variables qualitatives les plus pertinentes dans la discrimination des groupes concernaient la densité du tallage et l'intensité de la floraison.

Conclusion et recommandations : Les variables quantitatives permettant de mieux expliquer la diversité des clones présélectionnées au stade ligne de $3 \mathrm{~m}$ comprenaient, dans l'ordre décroissant, le poids moyen d'une tige, le diamètre moyen des tiges, la longueur moyenne des tiges, le nombre de tiges usinables $/ 3 \mathrm{~m}$ et le brix. Les variables qualitatives les plus pertinentes dans la discrimination des groupes concernaient la densité du tallage et l'intensité de la floraison La variabilité génétique ainsi révélée en canne vierge devra être vérifiée surtout en 1ère repousse avant la présélection définitive des clones pour les deux dernières étapes du processus de sélection variétale à conduire durant 5 ans en plantations commerciales.

Mots clés. Sélection massale, vigueur végétative, stade ligne, nouvelle plantation, variable quantitative, variable qualitative, analyse multivariée, groupe discriminé, adaptation, agro-écologie. 


\section{ABSTRACT \\ Genetic Variability of Sugarcane Genotypes Preselected at One-Row Stage as Plant Crop in Ferké, Northern Ivory Coast}

Objectives: The overall study objective was to contribute to sugarcane yield improvement in Ivory Coast. Specific objective was to evaluate the genetic diversity of sugarcane clones preselected at one-row stage as plant crop.

Methodology and results : It was conducted on Ferké 1 experimental station under full covering sprinkler irrigation in northern Ivory Coast. The genotypes were preselected as plant cane among 863 clones planted one raw of $3 \mathrm{~m} /$ clone following families, without replication and compared to control variety SP70/1006. Quantitative as well as qualitative traits observed were subjected to a series of multivariate analyses. It came out that quantitative traits which better explained diversity of genotypes were the following in decreasing order : average stalk weight, stalk diameter, number of millable stalks $/ 3 \mathrm{~m}$, stalk height and brix. Those phenotypic traits highly contributed to discrimination of varieties into 10 clusters which suggested a good genetic diversity among genotypes. Most relevant qualitative variables in the discrimination of clusters concerned tillering density and flowering intensity.

Conclusion and application of results : Quantitative traits most relevant in genotype clustering were average stalk weight, stalk diameter, number of millable stalks $/ 3 \mathrm{~m}$, stalk height and brix. Most relevant qualitative variables in genotype clustering concerned tillering density and flowering intensity. The genetic variability so shown in plant cane needs to be verified especially in 1 rst ratoon cane before final preselection of genotypes required for the last two stages of screening under commercial field conditions.

Keywords: visual selection, vegetative vigor, one-row stage, plant cane, phenotypic trait, multivariate analysis, clustering, adaptation, agro-ecology.

\section{INTRODUCTION}

La canne à sucre une culture vivrière économiquement importante dans les régions tropicales et subtropicales (Sivaneanesan and Waller, 1986). Elle est cultivée dans nombre de pays situés entre les latitudes $40^{\circ} \mathrm{N}$ et $32^{\circ} \mathrm{S}$ (Tomas, 2008) et le sucre de canne constitue environ $60 \%$ de la production mondiale de sucre (Amalraj and Balasundaram, 2009). La production mondiale de canne à sucre s'élève à 1700 millions de t/an, soit environ 1380 millions de t de sucre/an (FAO Stat, 2013). Les pays les plus gros producteurs de sucre de canne sont, dans l'ordre décroissant, le Brésil, l'Inde et la Chine avec respectivement 455, 281 et 100 millions de t/an. Cela représente, respectivement, 33, 23 et $7 \%$ de la production mondiale de sucre. La production africaine de canne à sucre est d'environ $5 \%$ de la production mondiale (85 millions de t/an, soit 8.5 millions de $t$ de sucre/an) dont $30 \%$ proviennent d'Afrique de l'Est (25.5 millions de t/an, soit 2.5 millions de $t$ de sucre/an). L'Afrique du Sud en est le 1 er producteur africain avec 20 millions de t/an, soit environ 2 millions de $t$ de sucre/an, suivie du Soudan, du
Kenya et du Swaziland. La production ivoirienne de canne à sucre s'élève à près de 2 millions de t/an, soit $0.1 \%$ de la production mondiale et $2.3 \%$ de la production africaine. La Côte d'Ivoire produit environ $200000 \mathrm{t}$ de sucre/an (soit $0.1 \%$ de la production sud-africaine) pour un besoin de consommation estimé à 240000 t/an. Avec une croissance démographique ivoirienne égale à $3.7 \% / a n$, les besoins en sucre de la population à l'horizon 2025 sont estimés à 320000 t/an (Zadi et al, 2016). Un tel niveau de production serait quasiment inatteignable à terme sans une amélioration significative de la compétitivité du secteur par l'accroissement de la productivité agricole en passant de 8.5-9 tà 10 voire $12 \mathrm{t}$ de sucre/ha. Pour ce faire, la politique agricole mise en œuvre avec le soutien de l'Union Européenne de 2009 à 2017 vise la diversification de la filière cannière (avec cogénération d'électricité à partir de la bagasse, production d'éthanol et de rhume), l'optimisation des pratiques culturales (travail du sol, gestion de la fertilité des sols, de l'eau d'irrigation et de l'enherbement) et l'adoption de variétés hautement productives en sucre adaptées 
aux conditions agro-écologiques locales (Péné, 2016). La sélection variétale de canne à sucre à partir des seedlings constitue un volet important de cette politique agricole. Elle complète celle basée sur la sélection de variétés commerciales ou présélectionnées introduites en Côte d'Ivoire à partir des quarantaines de Montpellier en France et de Réduit à l'lle Maurice, mise en œuvre depuis les années 1970 et qui se poursuit jusqu'à présent avec désormais l'acquisition de variétés protégées parallèlement à celles libres de droit. Dans un contexte de compétition internationale croissante, chaque pays producteur de canne à sucre chercheà améliorer ses rendements agricoles, dans une perspective de développement durable visant à maintenir le revenu des planteurs. L'augmentation des rendements passe souvent par la culture de nouvelles variétés plus productives qui procurent des gains de tonnages ainsi qu'une richesse saccharine supérieure. L'obtention de nouvelles variétés résultent de travaux de recherche de longue haleine visant à améliorer génétiquement la plante concernée. Le schéma général de sélection de la canne à sucre est typiquement de type clonal, semblable à celui de toute espèce de plante à multiplication végétative. Le principe est basé sur le

\section{MATÉRIEL ET MÉTHODES}

Site d'étude: L'étude a été menée à la station expérimentale du complexe sucrier de Ferké 1 , au Nord Côte d'Ivoire $\left(9^{\circ} 20^{\prime}-9^{\circ} 60^{\prime} \mathrm{N}, 5^{\circ} 22^{\prime}-5^{\circ} 40^{\prime} \mathrm{O}, 325 \mathrm{~m}\right)$. Le climat qui prévaut dans la zone est de type tropical sec caractérisé par deux saisons : l'une sèche, s'étend de novembre à avril et l'autre humide, de mai à octobre. La saison sèche est marquée par l'harmattan, un vent chaud et sec qui s'étend de mi-novembre à fin janvier. Le régime pluviométrique est de type unimodal, centré sur les mois d'aout-septembre qui cumulent presque la moitié de la hauteur moyenne annuelle de précipitations égale à 1200 $\mathrm{mm}$ avec une température moyenne journalière de $27^{\circ} \mathrm{C}$. Les températures minimale et maximale moyennes journalières sont respectivement de 32,5 et $21^{\circ} \mathrm{C}$. Pour satisfaire les besoins en eau de la canne à sucre, les apports par irrigation avoisinent les $700 \mathrm{~mm}$ par an (Péné et al, 2010). Le périmètre sucrier renferme majoritairement des sols peu profonds, formé sur granite ou gneiss. Les principales unités de sol sont de types ferralitique, hydromorphe de bas-fond et sableux des fait que toute nouvelle variété obtenue par hybridation sexuée est d'emblée fixée et reproductible à l'identique par clonage. II suffit donc de créer une variabilité génétique puis de choisir dans la population obtenue les meilleurs génotypes. Le schéma consiste donc à croiser entre eux des clones commerciaux ou des géniteurs élites, puis à sélectionner les clones-descendants les plus vigoureux, les meilleurs devenant de nouvelles variétés commerciales (Hoarau et al, 2002). Dans la sélection des seedlings de canne à sucre, la connaissance de la diversité génétique des variétés parentales est capitale car elle détermine le potentiel de chaque croisement pour l'effet d'hétérosis recherché et la fréquence des recombinaisons désirables dans les générations avancées (Gashaw, Mekbib and Ayana, 2016). De même, la distance génétique au sein du matériel parental joue un rôle essentiel car la diversité optimale de celui-ci est requise pour obtenir des génotypes performants dans les descendances à évaluer à travers un processus de sélection. La présente étude vise à évaluer la diversité génétique de 139 clones de canne à sucre présélectionnés en nouvelle plantation au stage ligne.

terrasses du fleuve Bandama. La texture du sol de la parcelle d'essai est sablo-argileuse.

Matériel végétal : Le matériel végétal présélectionné en nouvelle plantation (ou canne vierge) au stade ligne parmi 863 clones plantés par famille (sur un total de 39 familles) et comparés à un témoin d'origine brésilienne (SP701006), comprenait 139 individus ou clones (tableau 1). Ces clones présélectionnés, répartis en 29 familles, sont issus du 2ème lot de semences hybrides de canne à sucre importées en 2015 de La Réunion auprès du Centre Technique Sucrier (eRcane). Les semences sont issues de croisements bi-parentaux de variétés commerciales ou élites d'origines diversifiées (Réunion, Brésil, Australie, Soudan, Floride, Colombie, Afrique du Sud.).

Dispositif expérimental: Le dispositif expérimental adopté au stade ligne était un bloc incomplet, non randomisé comportant 863 clones plantés chacun sur une ligne de $3 \mathrm{~m}$ sans répétition excepté la variété témoin. Les clones, répartis en 39 familles, ainsi que le témoin ont 
été plantés en lignes simples (équidistantes de $1.5 \mathrm{~m}$ ) en novembre 2016 sur 11 blocs de $7 \mathrm{~m}$ de largeur et $30 \mathrm{~m}$ de longueur $\left(2310 \mathrm{~m}^{2}\right.$ utiles et $3210 \mathrm{~m}^{2}$ au total). Pour permettre une comparaison aisée des clones avec le témoin, celui-ci a été planté régulièrement tous les $7.5 \mathrm{~m}$ (après 5 individus). Pour limiter les effets de bordure, la parcelle d'essai a été ceinturée par une garde de $3 \mathrm{~m}$ de largeur et de $30 \mathrm{~m}$ de longueur constituée par une variété commerciale (R579).

Variables quantitatives et qualitatives observées : Les variables agro-morphologiques, phytosanitaires et technologiques observées en cours de végétation pour présélectionner les clones au stade ligne étaient les suivantes (tableau 2): nombre de tiges usinables sur $3 \mathrm{~m}$, diamètre de tige usinable, poids de tige usinable, brix (teneur en matière sèche dissoute dans le jus de canne), longueur de tige usinable, taux de floraison, absence de symptômes de maladies endémiques (charbon, pokkah boeng et échaudure foliaire), absence d'attaque sévère du foreur de tiges Eldana saccharina. Ces observations qui permettaient de déterminer la vigueur végétative des clones ont donné lieu à une note globale variant de 0 à 4 pour chacun des individus testés en comparaison avec la variété témoin. Le barème de notation était le suivant :

0 à 1 : Variétés à mauvaises aptitudes qui sont à éliminer ;

1,5: Variétés à repêcher, moins pourvues en certaines aptitudes ;

2 à 4 : Variétés à bonnes aptitudes $(\underline{3}$ : très bonnes aptitudes ; 4 : aptitudes exceptionnelles).

Les 139 variétés présélectionnées ont obtenu chacune une note globale supérieure 2 et étaient indemnes de symptômes de maladies endémiques (échaudure foliaire, charbon, pokkah boeng). À l'âge de 10 mois, les variables quantitatives suivantes ont été observées pour étudier la diversité de ces variétés : nombre de tiges usinables par ligne de $3 \mathrm{~m}$, diamètre de tige, longueur de tige usinable, poids de tige et brix réfractométrique. Les variables qualitatives observées concernaient la densité du tallage et l'intensité de la floraison.

Tableau 1 : Familles de clones plantés au stade " une ligne » la station expérimentale de Ferké 1. [Clone families planted at on-row stage in Ferké 1 experimental station]

\begin{tabular}{l|r|l|c|c}
\hline \multirow{2}{*}{ Famille } & \multicolumn{2}{|c|}{ Géniteurs } & \multicolumn{2}{c}{ Nombre d'individus (clones) } \\
\cline { 2 - 4 } & Femelle & Mâle & Total & présélectionnés en vierge \\
\hline F01 & R98/4009 & R95/4065 & 15 & 0 \\
F02 & N42 & R96/6422 & 4 & 1 \\
F03 & R92/2401 & R97/6375 & 42 & 1 \\
F04 & NC0 310 & R99/6153 & 28 & 7 \\
F05 & R03/4018 (e) & R04/8052 & 17 & 2 \\
F06 & R01/0277 & R95/2100 & 27 & 4 \\
F07 & RB83/5486 (e) & R575 & 161 & 33 \\
F08 & R81/0833 & SP70/1143 & 14 & 0 \\
F09 & N14 (e) & R585 & 46 & 10 \\
F10 & H32/8560 & R585 & 55 & 2 \\
F11 & R03/4018 (e) & N14 & 41 & 9 \\
F12 & R584 & R99/6153 & 19 & 10 \\
F13 & M1042/86 & PR83/1248 & 17 & 5 \\
F14 & R98/0814 & R585 & 14 & 2 \\
F15 & R96/2569 & R585 & 14 & 2 \\
F16 & H72/8597(e) & R585 & 26 & 1 \\
F17 & R582 & R585 & 20 & 5 \\
F18 & R83/0444 & N14 & 5 & 0 \\
F19 & R575 & N6 & 4 & 2 \\
F20 & R575 & CP81/1384 & 6 & 3 \\
F21 & R93/2351 & R99/6254 & 17 & 1 \\
F22 & R90/2992 & R97/2332 & 3 & 1 \\
F23 & R575 & R92/0804 & 6 & 3 \\
F24 & R91/4188 & R00/2460 & 10 & 1 \\
F25 & R94/6113 & R93/6769 & 16 & 4 \\
F26 & R92/6545 & R93/6683 & 11 & 3
\end{tabular}



Stade Ligne en Nouvelle Plantation à Ferké (Nord Côte d'Ivoire)

\begin{tabular}{l|r|l|c|c} 
F27 & R96/2569 & R97/2332 & 2 & 0 \\
F28 & R582 & R570 & 35 & 9 \\
F29 & R01/2072 & VMC71/238 & 7 & 0 \\
F30 & R93/0136 & R00/2460 & 14 & 2 \\
F31 & R89/2042 & R97/2332 & 10 & 0 \\
F32 & R11/7003 & N27 & 2 & 1 \\
F33 & R93/0136 & SP80/3280 & 2 & 0 \\
F34 & R579 & R94/6447 & 12 & 0 \\
F35 & R98/6095 & HoCP85/845 & 10 & 4 \\
F36 & TC9 & R95/4065 & 33 & 4 \\
F37 & R00/4009 & R95/4053 & 43 & 0 \\
F38 & R98/4009 & R98/4001 & 28 & 7 \\
F39 & RB83/5054 & R97/2335 & 27 & 0 \\
\hline \multicolumn{2}{|c|}{ Total } & 863 & 139 \\
\hline
\end{tabular}

Tableau 2 : Variables agro-morphologiques, phytosanitaires et technologiques ayant prévalu dans la pré-sélection des meilleurs clones en nouvelle plantation au stade ligne. [Agro-morphological, phytosanitary and technological variables used in pre-selecting best clones at one-row stage]

\begin{tabular}{l|l}
\hline Variables & Méthodes d'observation \\
\hline $\begin{array}{l}\text { Agro-morphologiques } \\
\text { Nombre de tiges } \\
\text { usinables/3m }\end{array}$ & Comptage du nombre de tiges usinables à 10 mois. \\
\hline Diamètre (mm) & $\begin{array}{l}\text { Mesure du diamètre de l'entre-nœud médian sur un échantillon représentatif de 10 } \\
\text { cannes usinables à l'aide d'un pied à coulisse. }\end{array}$ \\
\hline Tallage & Densité des talles observée à 3-4 mois (faible, moyen, très bon). \\
\hline Poids de tige usinable (kg) & Mesure du poids d'un échantillon de 10 cannes usinables par clone. \\
\hline \% Floraison & $\begin{array}{l}\text { Intensité de la floraison basée sur l'estimation du taux de floraison de chaque clone } \\
\text { (nombre de cannes ayant fleuri par rapport au nombre total de cannes par ligne à 10 } \\
\text { mois). }\end{array}$ \\
\hline Hauteur (m) & Mesure de la longueur moyenne d'un échantillon de 10 cannes usinables à 10 mois. \\
\hline Technologique & $\begin{array}{l}\text { Mesure au champ, à 10 mois, du taux de matière sèche dissoute dans le jus de canne } \\
\text { à l'aide d'un refractomètre portable. }\end{array}$ \\
\hline $\begin{array}{l}\text { Phytosanitaires } \\
\text { Maladies et ravageur } \\
\text { endémiques }\end{array}$ & $\begin{array}{l}\text { Absence de fouet charbonneux, absence de souche attaquée par l'échaudure foliaire et } \\
\text { le pokkha boeng. Absence d'attaque sévère du foreur de tiges (E. saccharina) }\end{array}$ \\
\hline
\end{tabular}

Analyses statistiques : Les logiciels Excel, Statistica version 7.1 et R 2.2 ont été utilisés pour l'analyse des données. La méthode d'analyse adoptée est basée sur la caractérisation des clones selon les caractères mesurés. Pour ce faire, les données obtenues ont dans un premier temps été saisies à l'aide du logiciel Excel sous la forme d'une base de données d'où un tableau croisé dynamique (TCD) a été réalisé. Celui-ci a permis d'extraire de ladite base de données les variables pertinentes à analyser qui sont les suivantes : nombre de tiges usinables, diamètre de tige usinable, longueur de tige usinable, brix, densité du tallage, intensité de floraison. Les logiciels Statistica et $\mathrm{R}$ ont par la suite été utilisés pour réaliser successivement trois méthodes d'analyses multivariées : analyse en composantes principales (ACP), classification ascendante hiérarchique $(\mathrm{CAH})$ et analyse factorielle discriminante (AFD). Les données ont été soumises au test $D^{2}$ de Mahalanobis pour mesurer les divergences génétiques entre différents groupes de clones à partir de leurs caractéristiques phénotypiques (Brasileiro et al, 2013 ; Duarte Filho et al, 2010, Chaudhary, 200/2001). Les données mesurées n'étant pas exprimées dans les mêmes échelles de mesure, il était nécessaire de les transformer en variables centrées-réduites comme 
rapporté par (Benzécri, 1970). La Classification Ascendante Hiérarchique (CAH) a ensuite été réalisée sur la base des distances euclidiennes entre les clones suivant la méthode de Ward comme option d'agrégation. Cette analyse multivariée a permis de structurer les variétés de canne présélectionnées selon leurs caractéristiques agro-morphologiques et technologiques.

\section{RÉSULTATS}

Dispersion des variables quantitatives observées à la récolte : Le nombre de tiges usinables par ligne de $3 \mathrm{~m}$ et le poids moyen d'une tige ont été les caractères quantitatifs les plus dispersés, avec $20 \%$ de coefficient de variation. Cela suggère que ces variables contribuaient fortement dans la diversité génétique des
L'AFD a été réalisée enfin pour évaluer à l'aide du test $\lambda$ Wilks au seuil de $5 \%$ la robustesse des groupes précédemment formés à partir de la $\mathrm{CAH}$. Elle permet de vérifier l'appartenance des individus aux groupes précédemment constitués à l'aide de la CAH (Volle, 1997).

clones étudiées. Les trois autres variables quantitatives observées (diamètre de tige, longueur de tige et brix réfractométrique) présentaient chacune une dispersion d'environ $10 \%$ (tableau 3). Celles-ci devraient contribuer à un degré moindre à la diversité génétique de ces clones présélectionnés.

Tableau 3. Moyennes des variables agro-morphologiques et technologiques observées à la récolte sur 139 clones présélectionnés en vierge et leurs dispersions respectives. [Means of agro-morphological and technological traits observed at harvest on 139 preselected genotypes and their respective dispersion]

\begin{tabular}{l|c|c|c|c}
\hline Variable quantitative & Minimum & Maximum & Moyenne & CV (\%) \\
\hline Tall. / 3m (-) & 20 & 92 & 51 & 20 \\
Diamètre (mm) & 18,3 & 36,0 & 25,8 & 10 \\
Longueur (m) & 2,20 & 4,24 & 3,15 & 10 \\
Poids (kg) & 0,7 & 2,5 & 1,5 & 20 \\
Brix champ (-) & 7,4 & 23,4 & 18,5 & 10 \\
\hline
\end{tabular}

CV : coefficient de variation

Analyse en composantes principales: Les cinq couples de variables les mieux corrélés sont DiamètreNombre de tiges/3m, poids d'une tige-longueur d'une tige, poids d'une tige-diamètre, Brix-Nombre de tiges/3m et Longueur d'une tige-Diamètre, avec des coefficients respectifs de $0.84,-0.80,0.78,0.58$ et 0.57 (tableau 4). Les plus faiblement corrélées sont par ordre croissant en valeur absolue Longueur d'une tige- $\mathrm{Nb}$ de tiges $/ 3 \mathrm{~m}$ $(0.00)$, Brix-Longueur de tiges (-0.20), Brix-Diamètre
(0.30), Brix-Poids moyen d'une tige $(-0.32)$ et Poids moyen d'une tige-Nombre de tiges/3 m (-0.32). II ressort de l'ACP que les variables permettant mieux expliquer la diversité des variétés présélectionnées sont, dans l'ordre décroissant, le poids moyen d'une tige, le diamètre moyen d'une tige, la longueur moyenne d'une tige et le nombre de tiges $/ 3 \mathrm{~m}$. Le Brix est par contre la variable quantitative expliquant le moins la diversité des clones présélectionnés (figures 1 et 2, tableau 5).

Tableau 4. Matrice de corrélations entre les caractères quantitatifs observés à la récolte chez les 139 clones présélectionnées au stade ligne à Ferké. [Correlation matrix of quantitative traits observed at harvest on 139 clones at one-row stage in Ferké]

\begin{tabular}{l|r|r|r|r|r}
\hline & Nb tiges/3m & \multicolumn{1}{|c|}{ Diamètre } & Longueur & \multicolumn{1}{|c|}{ Poids } & Brix au champ \\
\hline Nb tiges/3m & 1,00 & & & & \\
Diamètre tige & $-\mathbf{0 , 8 0}$ & 1,00 & & & \\
Longueur tige & 0,00 & $\mathbf{0 , 5 7}$ & 1,00 & & \\
Poids d'une tige & $-0,32$ & $\mathbf{0 , 7 8}$ & $\mathbf{0 , 8 4}$ & 1,00 & \\
Brix au champ & $-\mathbf{0 , 5 8}$ & 0,30 & $-0,20$ & $-0,32$ & 1,00 \\
\hline
\end{tabular}

En gras, les coefficients de corrélation supérieurs à 0,5 en valeur absolue excepté la corrélation parfaite 1,0. [In bold : correlation oefficients higher that 0.5 in absolute value except for perfect correlation 1.0] 


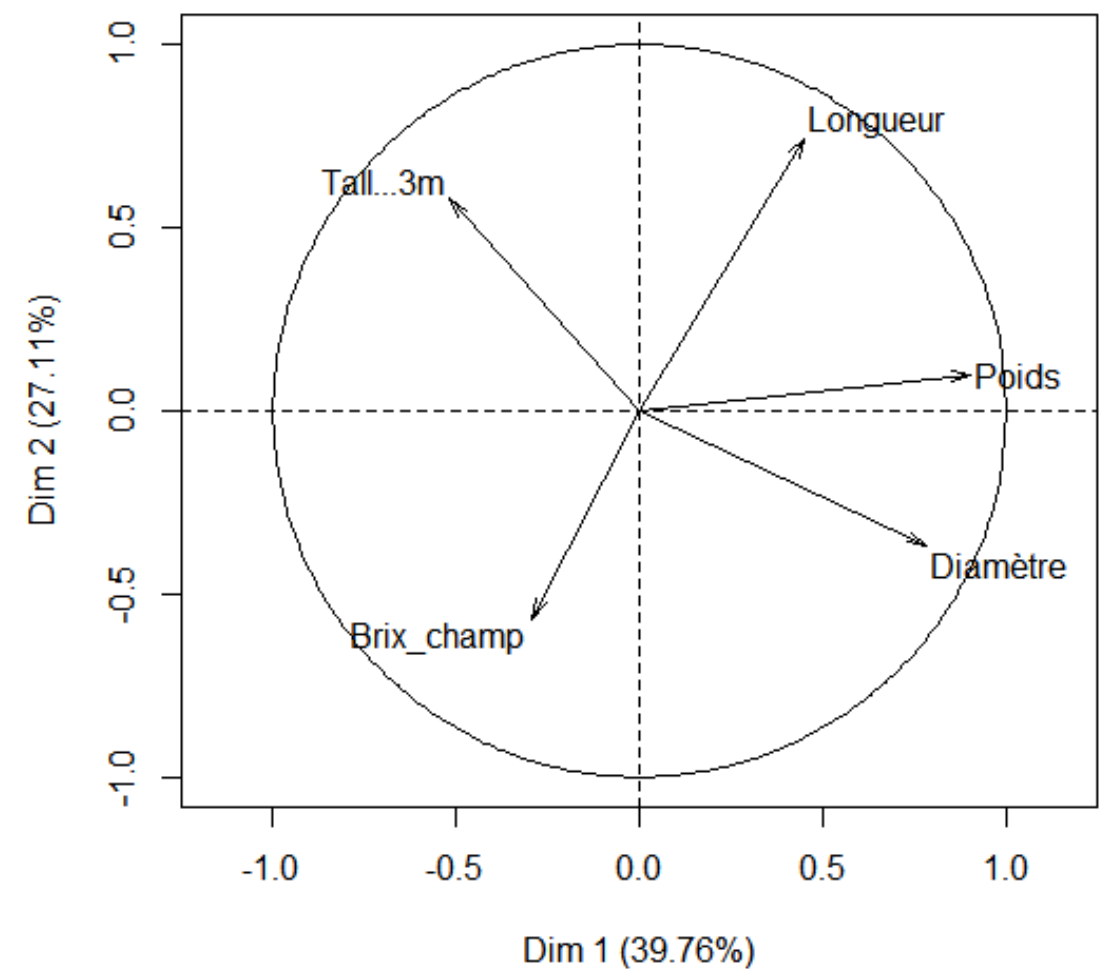

Figure 1: Cercle de corrélations résultant de la projection des clones et des variables agro-morphologiques et technologiques observées dans le plan factoriel 1-2 selon l'ACP. [Correlation circle resulting from projection of clones and agro-morphological traits observed in 1-2 factor plan following PCA]

Tableau 5. Numéros des clones présélectionnés en canne vierge au stade ligne pour l'ACP.

\begin{tabular}{l|l|l|l|l|l|l|l|l|l}
\hline $\mathbf{N}^{\circ}$ & Clone & $\mathbf{N}^{\circ}$ & Clone & $\mathbf{N}^{\circ}$ & Clone & $\mathbf{N}^{\circ}$ & Clone & $\mathbf{N}^{\circ}$ & Clone \\
\hline 1 & F02_L02 & 31 & F07_L103 & 61 & F11_L05 & 91 & F17_L06 & 121 & F28_L32 \\
2 & F03_L28 & 32 & F07_L105 & 62 & F11_L06 & 92 & F17_L07 & 122 & F30_L11 \\
3 & F04_L11 & 33 & F07_L110 & 63 & F11_L11 & 93 & F17_11 & 123 & F30_L12 \\
4 & F04_L13 & 34 & F07_L112 & 64 & F11_L17 & 94 & F17_L18 & 124 & F32_L02 \\
5 & F04_L14 & 35 & F07_L132 & 65 & F11_L25 & 95 & F19_L02 & 125 & F35_L03 \\
6 & F04_L15 & 36 & F07_L133 & 66 & F11_L30 & 96 & F19_04 & 126 & F35_L05 \\
7 & F04_L16 & 37 & F07_L134 & 67 & F11_L31 & 97 & F20_L01 & 127 & F35_L06 \\
8 & F04_L17 & 38 & F07_L140 & 68 & F11_L37 & 98 & F20_L02 & 128 & F35_L10 \\
9 & F04_L23 & 39 & F07_L142 & 69 & F11_L40 & 99 & F20_L04 & 129 & F36_L03 \\
10 & F05_L02 & 40 & F07_L143 & 70 & F12_L02 & 100 & F21_L03 & 130 & F36_L10 \\
11 & F05_L17 & 41 & F07_L146 & 71 & F12_L03 & 101 & F22_02 & 131 & F36_L17 \\
12 & F06_L05 & 42 & F07_L149 & 72 & F12_L05 & 102 & F23_L01 & 132 & F36_L25 \\
13 & F06_L10 & 43 & F07_L151 & 73 & F12_L07 & 103 & F23_L04 & 133 & F38_L15 \\
14 & F06_L19 & 44 & F07_L153 & 74 & F12_L08 & 104 & F23_05 & 134 & F38_L16 \\
15 & F06_L23 & 45 & F07_L154 & 75 & F12_L12 & 105 & F24_L05 & 135 & F38_L20 \\
16 & F07_L03 & 46 & F07_L155 & 76 & F12_L14 & 106 & F25_L01 & 136 & F38_L22 \\
17 & F07_L07 & 47 & F07_L158 & 77 & F12_L16 & 107 & F25_L10 & 137 & F38_L24 \\
18 & F07_L09 & 48 & F07_L159 & 78 & F12_L18 & 108 & F25_L12 & 138 & F38_L27 \\
19 & F07_L10 & 49 & F09_L15 & 79 & F12_L19 & 109 & F25_14 & 139 & F38_L28 \\
20 & F07_L12 & 50 & F09_L18 & 80 & F13_L02 & 110 & F26_L05 & &
\end{tabular}



Stade Ligne en Nouvelle Plantation à Ferké (Nord Côte d'Ivoire)

\begin{tabular}{l|l|l|l|l|l|l|l|l}
21 & F07_L15 & 51 & F09_L20 & 81 & F13_L03 & 111 & F26_L06 & \\
22 & F07_L24 & 52 & F09_L22 & 82 & F13_L04 & 112 & F26_L07 & \\
23 & F07_L35 & 53 & F09_L23 & 83 & F13_L06 & 113 & F28_08 & \\
24 & F07_L45 & 54 & F09_L29 & 84 & F13_L09 & 114 & F28_L09 & \\
25 & F07_L53 & 55 & F09_L31 & 85 & F14_L02 & 115 & F28_L10 & \\
26 & F07_L59 & 56 & F09_L34 & 86 & F14_L12 & 116 & F28_L11 & \\
27 & F07_L67 & 57 & F09_L43 & 87 & F15_L05 & 117 & F28_L12 & \\
28 & F07_L73 & 58 & F09_L46 & 88 & F15_L07 & 118 & F28_L13 & \\
29 & F07_L79 & 59 & F10_L04 & 89 & F16_L01 & 119 & F28_L18 & \\
30 & F07_L95 & 60 & F10_L22 & 90 & F17_L04 & 120 & F28_L24 & \\
\hline
\end{tabular}

ACP : Analyse en composantes principales. [PCA : Principal Component Analysis]

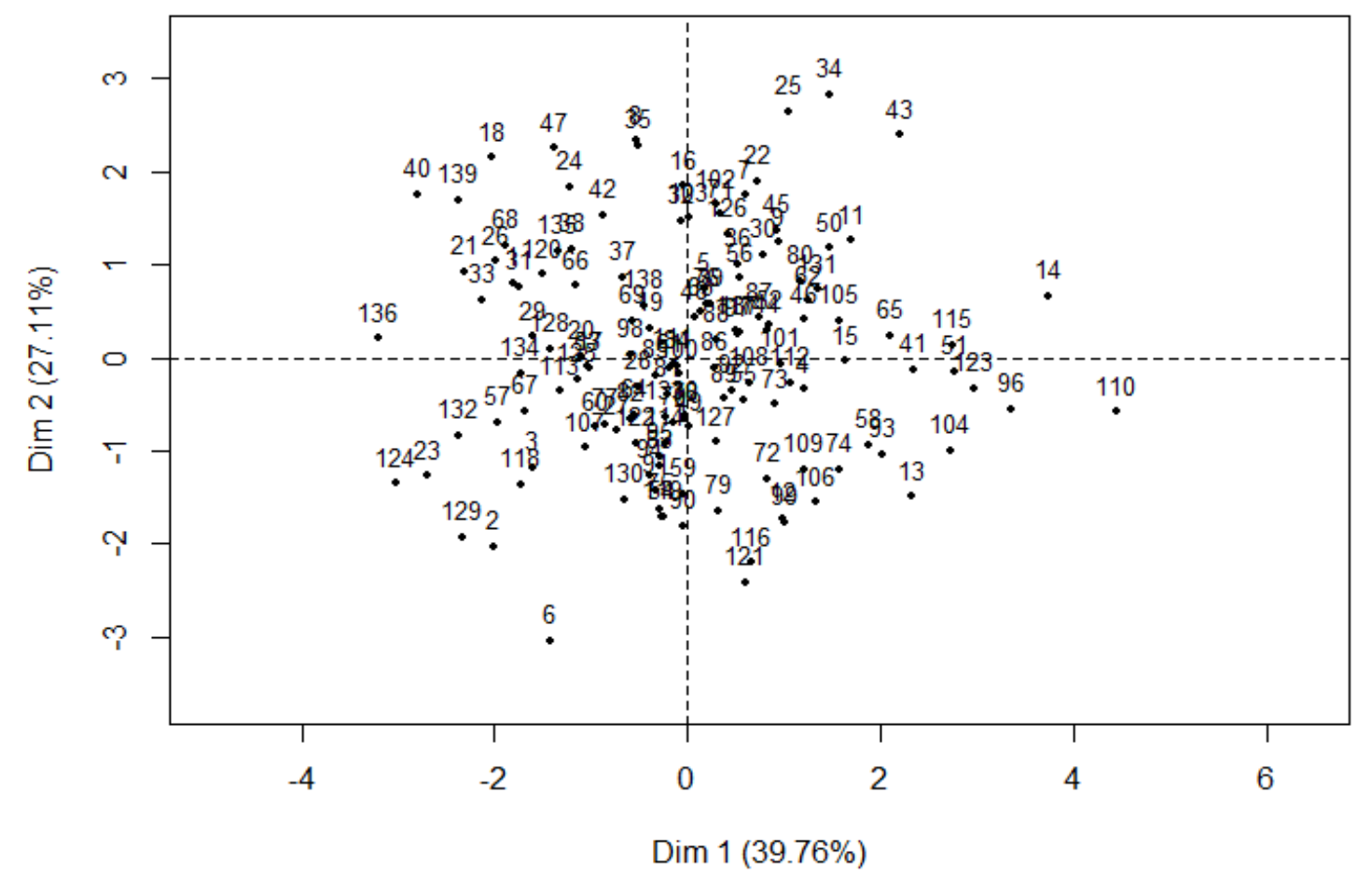

Figure 2 : Projection des 139 clones présélectionnés dans le plan factoriel 1-2 selon l'ACP basée sur la corrélation des caractères quantitatifs observés à la récolte. [Projection of 139 clones in 1-2 factor plan following PCA based on correlation of quantitative traits observed at harvest]

Classification ascendante hiérarchique et analyse factorielle discriminante : Le dendrogramme résultant de la classification ascendante hiérarchique (CAH) basée sur la méthode de Ward, met en évidence 10 groupes de variétés pour un niveau de troncature égal à 6 (figure 2). Cela suggère une assez bonne diversité génétique au sein des 139 individus présélectionnés. L'analyse factorielle discriminante (AFD) montre que ces 10 groupes de variétés sont significativement dissemblables $(p<0.00001)$, (tableau 6). Le tableau 7 présente le nombre et la liste nominative des clones composant chaque groupe déterminé. Les groupes G3, G2, G6 et G4 étaient les mieux représentés (56\% de l'effectif total) avec respectivement 23, 21, 19 et 1514 individus. Les groupes $\mathrm{G} 7, \mathrm{G} 8, \mathrm{G} 10$ et $\mathrm{G} 9$ étaient les moins représentés $(24.5 \%)$ avec respectivement $11,9,8$ et 6 individus. La moyenne relative à chacune des variables quantitatives observées à la récolte est donnée dans le tableau 8. Celui-ci montre que les clones issus des groupes $\mathrm{G} 1$ et G6 ont le plus fort tallage, avec respectivement 76 et 60 tiges usinables $/ 4.5 \mathrm{~m}^{2}$ (environ 169000 et 133000 tiges/ha), tandis que ceux issus de G5, G8, G9 et G10 se distinguent par leur faible tallage, avec en moyenne 38 à 40 tiges usinables $/ 4.5 \mathrm{~m}^{2}$ soit environ 84000 à 89000 
tiges/ha. Les clones à fort diamètre sont issus des groupes G9 et G8 avec en moyenne 32.9 et $30.6 \mathrm{~mm}$ respectivement, et ceux à faible diamètre des groupes $\mathrm{G} 7, \mathrm{G} 1$ et $\mathrm{G} 5$, avec en moyenne 21.0 à $23.5 \mathrm{~mm}$. Les clones à fort poids moyen d'une tige sont issus des groupes G9, G10 et G8 avec 1.8 à $2.3 \mathrm{~kg} /$ tige et ceux à faible poids moyen de G5, G1 et G2 avec 1.1 à 1.3 $\mathrm{kg} /$ tige. Les individus à tiges longues sont issus des groupes $\mathrm{G} 6$ et $\mathrm{G} 10$ avec 3.5 à $3.7 \mathrm{~m}$ en moyenne et ceux à tiges petites de $\mathrm{G} 5$ et $\mathrm{G} 2$ avec 2.7 à $2.8 \mathrm{~m}$ en moyenne. Enfin, les clones à brix élevé sont issus des groupes $\mathrm{G} 2$, G7, G8 et G3 avec 20 à 20.8 en moyenne et ceux à faible brix de G4, G6 et G9, soit 13.8 à 16.9. II ressort de l'analyse factorielle des correspondances (AFC) que les clones du groupe $\mathrm{G} 1$ ont un taux de floraison élevé et un tallage excellent ou très bon (figure 4). De tels niveaux de tallage qui conviennent bien pour la plantation en simples rangs sont réputés être associés à un bon rendement en canne et une bonne tenue en repousse de la canne à sucre. Quant au taux de floraison élevé, il caractérise les variétés à cultiver préférentiellement en début de campagne de récoltes (octobre à décembre). Ceux issus des groupes G2, G3, G4 et G6 ont un tallage bon et un faible taux de floraison. Les clones des groupes G5, G7, G8 et G10 tendent à présenter un tallage faible à moyen et un très faible taux de floraison. Ces derniers clones conviendraient pour la plantation en rangs jumelés (pour compenser le faible tallage) et la culture en fin de campagne de récoltes (février à avril). Sur les 139 clones présélectionnés en canne vierge (tableau 9), la famille F07 était la plus fortement représentée avec 33 individus (23.7\% du total). Elle intervient dans la formation de 7 groupes sur les 10 qui ont été déterminés (G1, G3, G4, G5, G6, G7 et G10). Elle est particulièrement représentée dans les groupes G7 (54.5\%), G1 (50\%), G6 (47.4\%), G10 $(37.5 \%)$ et G4 $(26.7 \%)$. Elle est suivie des familles $\mathrm{F} 09, \mathrm{~F} 12, \mathrm{~F} 11$ et $\mathrm{F} 28$ avec chacune 9 à 10 individus (6.5 à $7 \%$ du total). Ces 5 familles citées sont représentées par un total de 71 clones, soit $51 \%$ des clones présélectionnés.

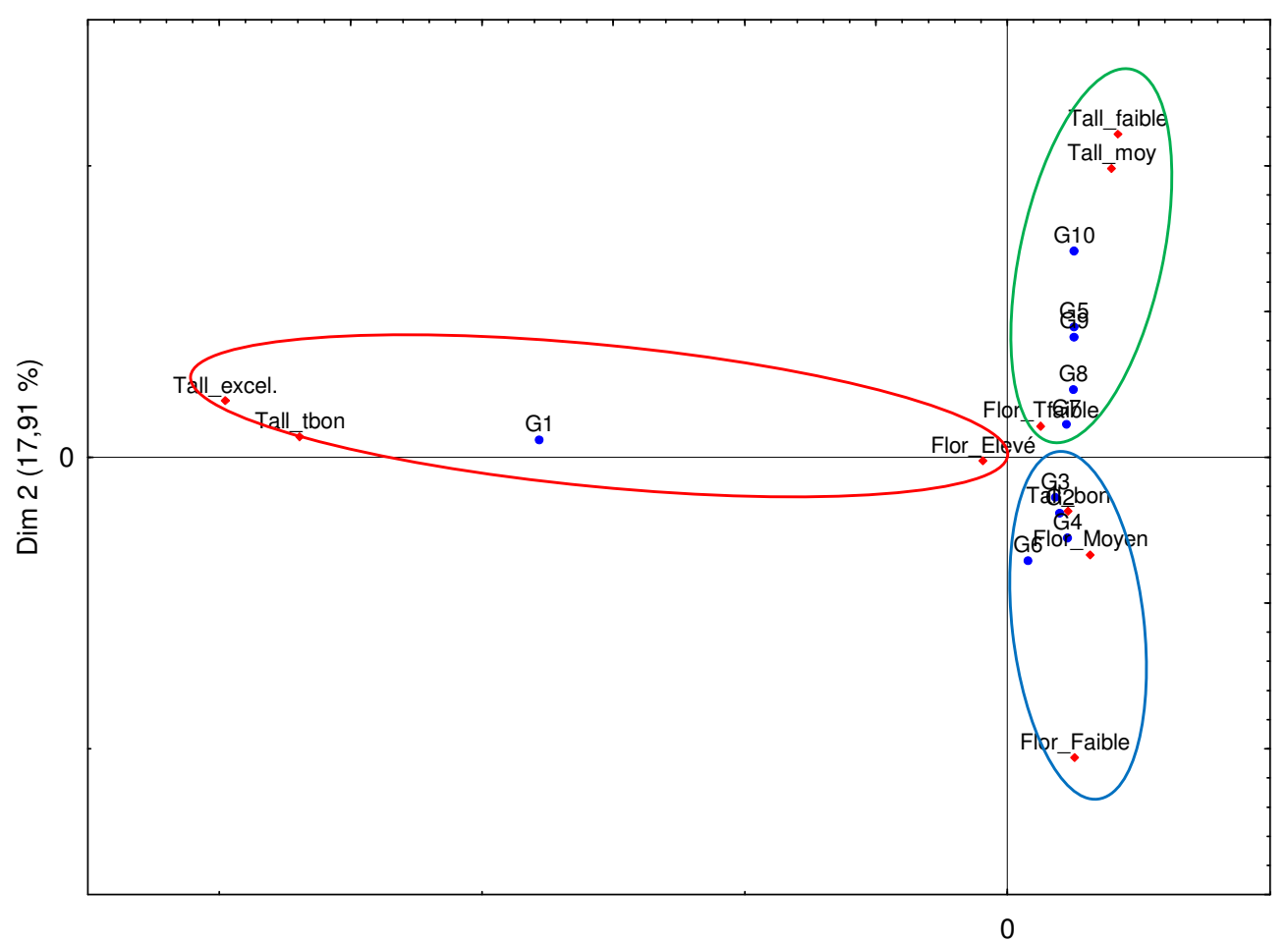

$\operatorname{Dim} 1(68,24 \%)$

Figure 4 : Projection des différents groupes de clones déterminés par CAH et des caractères qualitatifs observés à la récolte dans le plan factoriel 1-2 formé par l'AFC. [Projection of different clusters and qualitative traits in 1-2 factor plan following correspondence factor analysis - CFA] 


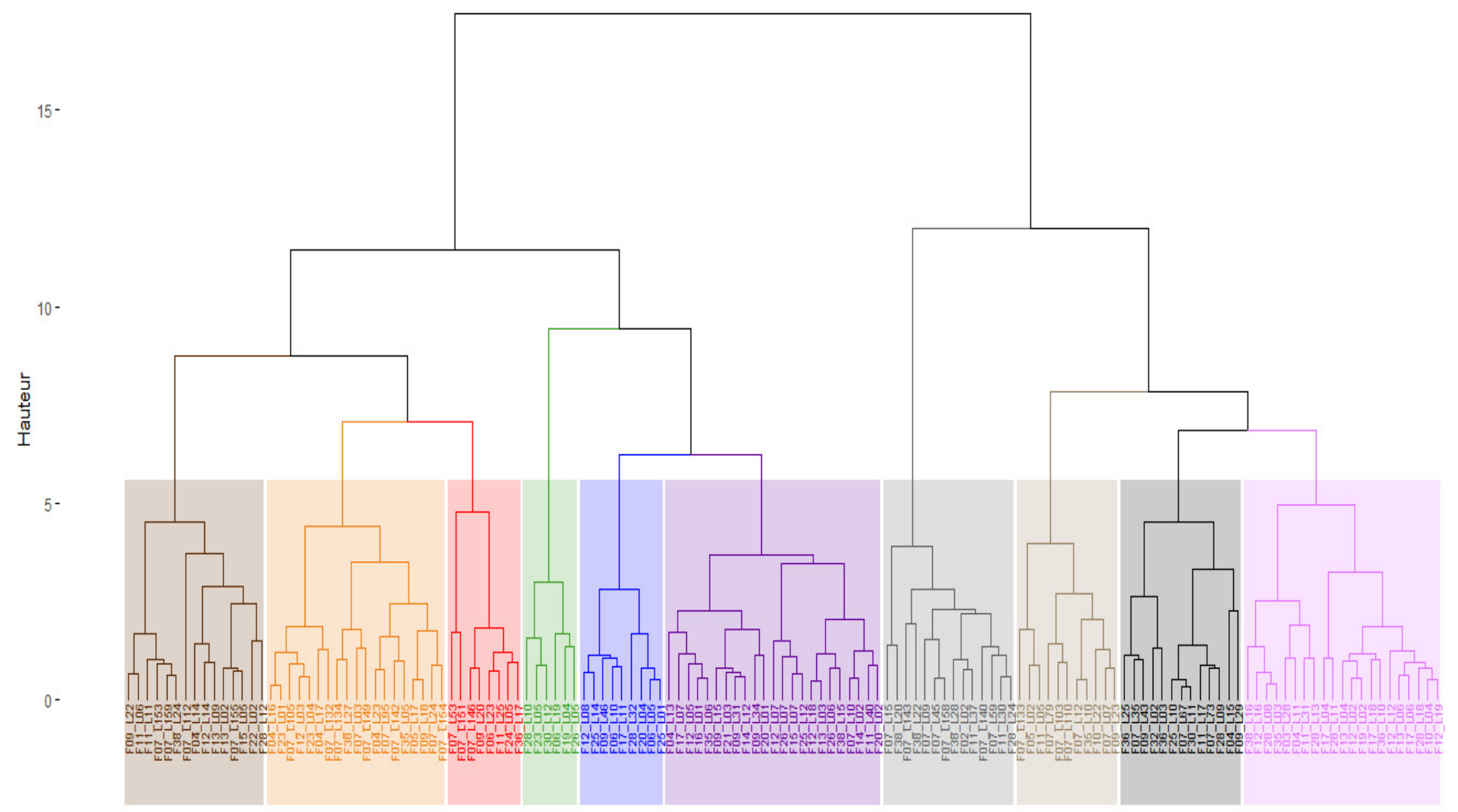

Figure 3. Dendrogramme résultant de la CAH relative aux 139 clones présélectionnés répartis en 10 groupes distincts. [Dendrogram resulted from cluster analysis regarding 139 cane genotypes split into 10 different groups] 
Béhou et al, J. Appl. Biosci. 2018 Variabilité Génétique des Clones de Canne à Sucre Présélectionnées au Stade Ligne en Nouvelle Plantation à Ferké (Nord Côte d'Ivoire)

Tableau 6 : Distance de Mahalanobis au carré entre les 10 groupes de clones de canne à sucre déterminés par CAH et test de Fisher associé. [Mahalanobis distance square between clusters taken 2 by 2 and associated Fisher test]

\begin{tabular}{l|c|c|c|c|c|c|c|c|c|c}
\hline & Groupe 1 & Groupe 2 & Groupe 3 & Groupe 4 & Groupe 5 & Groupe 6 & Groupe 7 & Groupe 8 & Groupe 9 & Groupe 10 \\
\hline Groupe 1 & - & $\mathrm{F}=31,50$ & $\mathrm{~F}=28,74$ & $\mathrm{~F}=37,69$ & $\mathrm{~F}=39,04$ & $\mathrm{~F}=18,58$ & $\mathrm{~F}=24,97$ & $\mathrm{~F}=46,34$ & $\mathrm{~F}=53,80$ & $\mathrm{~F}=41,85$ \\
Groupe 2 & 19,351 & - & $\mathrm{F}=10,17$ & $\mathrm{~F}=39,79$ & $\mathrm{~F}=17,66$ & $\mathrm{~F}=41,31$ & $\mathrm{~F}=20,85$ & $\mathrm{~F}=12,52$ & $\mathrm{~F}=39,10$ & $\mathrm{~F}=36,42$ \\
Groupe 3 & 17,038 & 4,780 & - & $\mathrm{F}=27,08$ & $\mathrm{~F}=31,05$ & $\mathrm{~F}=16,42$ & $\mathrm{~F}=17,34$ & $\mathrm{~F}=9,02$ & $\mathrm{~F}=23,44$ & $\mathrm{~F}=16,11$ \\
Groupe 4 & 26,856 & 23,465 & 15,391 & - & $\mathrm{F}=26,10$ & $\mathrm{~F}=17,78$ & $\mathrm{~F}=22,99$ & $\mathrm{~F}=29,86$ & $\mathrm{~F}=26,33$ & $\mathrm{~F}=16,99$ \\
Groupe 5 & 29,882 & 11,346 & 19,288 & 19,335 & - & $\mathrm{F}=48,33$ & $\mathrm{~F}=11,97$ & $\mathrm{~F}=31,47$ & $\mathrm{~F}=52,79$ & $\mathrm{~F}=39,48$ \\
Groupe 6 & 11,896 & 21,369 & 8,144 & 10,943 & 32,311 & - & $\mathrm{F}=20,98$ & $\mathrm{~F}=31,02$ & $\mathrm{~F}=27,61$ & $\mathrm{~F}=132,03$ \\
Groupe 7 & 20,918 & 14,902 & 12,027 & 18,692 & 10,369 & 15,536 & - & $\mathrm{F}=27,88$ & $\mathrm{~F}=41,61$ & $\mathrm{~F}=17,74$ \\
Groupe 8 & 43,652 & 10,255 & 7,196 & 27,392 & 30,534 & 26,205 & 29,065 & - & $\mathrm{F}=10,75$ & $\mathrm{~F}=17,27$ \\
Groupe 9 & 66,092 & 41,020 & 25,420 & 31,698 & 66,352 & 31,247 & 55,303 & 15,414 & - & $\mathrm{F}=13,27$ \\
Groupe 10 & 42,415 & 32,435 & 14,009 & 16,801 & 41,131 & 11,031 & 19,764 & 21,038 & 19,965 & - \\
\hline P<0,003
\end{tabular}

$P<0,00001$ pour toutes les valeurs de $F$ 
Tableau 7 : Clones de canne à sucre composant les différents groupes déterminés par CAH.[Cane genotypes composing different clusters determined]

\begin{tabular}{|c|c|c|}
\hline Groupe & Nombre de clones & Liste des clones \\
\hline G1 & 14 & $\begin{array}{l}\text { F02_L02, F07_L09, F07_L15, F07_L45, F07_L59, F07_L140, F07_L143, } \\
\text { F07_L158, F11_L30, F11_L37, F28_L24, F38_L20, F38_L22, F38_L28 }\end{array}$ \\
\hline G2 & 21 & $\begin{array}{l}\text { F03_L28, F04_L11, F10_L04, F11_L31, F12_L02, F12_L12, F12_L16, } \\
\text { F12_L19, F13_L04, F13_L06, F17_L04, F17_L06, F17_L18, F19_L02, } \\
\text { F28_L08, F28_L11, F28_L13, F28_L18, F35_L03, F36_L10, F38_L16 }\end{array}$ \\
\hline G3 & 23 & $\begin{array}{l}\text { F04_L13, F07_L10, F09_L15, F09_L31, F09_L34, F11_L40, F12_L05, } \\
\text { F12_L07, F12_L18, F13_L03, F14_L02, F14_L12, F15_L07, F16_L01, } \\
\text { F17_L07, F20_L01, F20_02, F21_L03, F25_L12, F26_L06, F26_L07, } \\
\text { F35_L06, F38_L15 }\end{array}$ \\
\hline G4 & 15 & $\begin{array}{l}\text { F04_L14, F07_L112, F07_L153, F07_L155, F07_L159, F09_L22, F11_L06, } \\
\text { F11_L11, F12_L14, F13_L02, F13_L09, F15_L05, F22_L02, F28_L12, } \\
\text { F38_L24 }\end{array}$ \\
\hline G5 & 13 & $\begin{array}{l}\text { F04_L15, F07_L35, F07_L67, F07_L73, F09_L29, F09_L43, F11_L17, } \\
\text { F25_L10, F28_L09, F30_L11, F32_L02, F36_L03, F36_L25 }\end{array}$ \\
\hline G6 & 19 & $\begin{array}{l}\text { F04_L16, F04_L17, F04_L23, F05_L17, F07_L03, F07_L24, F07_L95, } \\
\text { F07_L105, F07_L132, F07_L134, F07_L142, F07_L149, F07_L154, F09_L18, } \\
\text { F12_L03, F23_L01, F23_L04, F35_L05, F38_L27 }\end{array}$ \\
\hline G7 & 11 & $\begin{array}{l}\text { F05_L02, F07_L07, F07_L12, F07_L79, F07_L103, F07_L110, F07_L133, } \\
\text { F09_L23, F10_L22, F11_L05, F35_L10 }\end{array}$ \\
\hline G8 & 9 & $\begin{array}{l}\text { F06_L05, F06_L10, F09_L46, F12_L08, F17_L11, F20_L04, F25_L01, } \\
\text { F25_L14, F28_L32 }\end{array}$ \\
\hline G9 & 6 & F06_L19, F19_L04, F23_L05, F26_L05, F28_L10, F30_L12 \\
\hline G10 & 8 & $\begin{array}{l}\text { F06_L23, F07_L53, F07_L146, F07_L151, F09_L20, F11_L25, F24_L05, } \\
\text { F36_L17 }\end{array}$ \\
\hline
\end{tabular}

Tableau 8: Valeurs moyennes des 10 groupes de clones de canne à sucre déterminés selon les 5 variables quantitatives observées à la récolte. [Means of all 10 cluster genotypes determined following five quantitative variables observed at harvest]

\begin{tabular}{|c|c|c|c|c|c|c|c|c|c|c|c|}
\hline & Moyenne & $\begin{array}{l}\text { Groupe } \\
1\end{array}$ & \begin{tabular}{|l|} 
Groupe \\
2
\end{tabular} & $\begin{array}{l}\text { Groupe } \\
3\end{array}$ & $\begin{array}{l}\text { Groupe } \\
4\end{array}$ & \begin{tabular}{|l|} 
Groupe \\
5
\end{tabular} & $\begin{array}{l}\text { Groupe } \\
6\end{array}$ & \begin{tabular}{|l} 
Groupe \\
7
\end{tabular} & \begin{tabular}{|l|} 
Groupe \\
8
\end{tabular} & \begin{tabular}{|l} 
Groupe \\
9
\end{tabular} & $\begin{array}{l}\text { Groupe } \\
10\end{array}$ \\
\hline $\begin{array}{l}\mathrm{Nb} \\
\text { tiges/3 m } \\
\text { Diamètre } \\
(\mathrm{mm}) \\
\text { Longueu } \\
\mathrm{r}(\mathrm{m}) \\
\text { Poids/tig } \\
\mathrm{e}(\mathrm{kg}) \\
\text { Brix au } \\
\text { champ }\end{array}$ & $\begin{array}{l}51 \\
25,76 \\
3,15 \\
1,50 \\
18,5\end{array}$ & $\begin{array}{l}76 \\
22,36 \\
3,13 \\
1,20 \\
18,1\end{array}$ & $\begin{array}{l}51 \\
26,53 \\
2,82 \\
1,35 \\
21,0\end{array}$ & $\begin{array}{l}51 \\
26,82 \\
3,19 \\
1,57 \\
20,0\end{array}$ & $\begin{array}{l}44 \\
26,73 \\
3,19 \\
1,47 \\
13,8\end{array}$ & $\begin{array}{l}40 \\
23,48 \\
2,74 \\
1,07 \\
18,9\end{array}$ & $\begin{array}{l}60 \\
24,94 \\
3,50 \\
1,61 \\
16,9\end{array}$ & $\begin{array}{l}46 \\
20,97 \\
3,23 \\
1,36 \\
20,2\end{array}$ & $\begin{array}{l}39 \\
30,61 \\
3,01 \\
1,81 \\
20,8\end{array}$ & $\begin{array}{l}40 \\
32,90 \\
3,25 \\
2,31 \\
16,2\end{array}$ & $\begin{array}{l}38 \\
26,35 \\
3,70 \\
1,93 \\
18,0\end{array}$ \\
\hline
\end{tabular}


Tableau 9 : Effectifs des clones de canne à sucre présélectionnées en vierge au stade ligne selon les familles et les groupes discriminés par la CAH. [Total number of preselected cane genotypes following different families and clusters]

\begin{tabular}{|c|c|c|c|c|c|c|c|c|c|c|c|}
\hline \multirow[b]{2}{*}{ Familles } & \multicolumn{11}{|c|}{ Groupes de clones discriminés } \\
\hline & G1 & G2 & G3 & G4 & G5 & G6 & G7 & G8 & G9 & G10 & Total \\
\hline H32/8560 x R585 (F10) & & 1 & 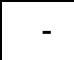 & - & - & - & 1 & - & - & - & 2 \\
\hline H72/8597 (e) x R585 (F16) & - & - & 1 & - & - & - & - & - & - & - & 1 \\
\hline M1042/86 x PR83/1248 (F13) & - & 2 & 1 & 2 & - & - & - & - & - & - & 5 \\
\hline N14 (e) x R585 (F09) & - & - & 3 & 1 & 2 & 1 & 1 & 1 & - & 1 & 10 \\
\hline N42 x R96/6422 (F02) & 1 & - & - & - & - & - & - & - & - & - & 1 \\
\hline NCo310 x R99/6153 (F04) & - & 1 & 1 & 1 & 1 & 3 & - & - & - & - & 7 \\
\hline R01/0277 x R95/2100 (F06) & - & - & - & - & - & - & - & 2 & 1 & 1 & 4 \\
\hline $\mathrm{R} 03 / 4018$ (e) x N14 (F11) & 2 & 1 & 1 & 2 & 1 & - & 1 & - & - & 1 & 9 \\
\hline $\mathrm{R} 03 / 4018$ (e) x R04/8052 (F05) & - & - & - & - & - & 1 & 1 & - & - & - & 2 \\
\hline R11/7003 x N27 (F32) & - & - & - & - & 1 & - & - & - & - & - & 1 \\
\hline R575 x CP81/1384 (F20) & - & - & 2 & - & - & - & - & 1 & - & - & 3 \\
\hline R575 x N6 (F19) & - & 1 & - & - & - & - & - & - & 1 & - & 2 \\
\hline R579 x R92/0804 (F23) & - & - & - & - & - & 2 & - & - & 1 & - & 3 \\
\hline R582 x R570 (F28) & 1 & 4 & - & 1 & 1 & - & - & 1 & 1 & - & 9 \\
\hline R582 x R585 (F17) & - & 3 & 1 & - & - & - & - & 1 & - & - & 5 \\
\hline R584 x R99/6153 (F12) & - & 4 & 3 & 1 & - & 1 & - & 1 & - & - & 10 \\
\hline R90/2992 x R97/2332 (F22) & - & - & - & 1 & - & - & - & - & - & - & 1 \\
\hline R91/4188 x R00/2460 (F24) & - & - & - & - & - & - & - & - & - & 1 & 1 \\
\hline R92/2401 x R97/6375 (F03) & - & 1 & - & - & - & - & - & - & - & - & 1 \\
\hline R92/6545 x R93/6683 (F26) & - & - & 2 & - & - & - & - & - & 1 & - & 3 \\
\hline R93/0136 x R00/2460 (F30) & - & - & - & - & 1 & - & - & - & 1 & - & 2 \\
\hline R93/2351 x R99/6254 (F21) & - & - & 1 & - & - & - & - & - & - & - & 1 \\
\hline $\mathrm{R} 94 / 6113$ x R93/6769 (F25) & - & - & 1 & - & 1 & - & - & 2 & - & - & 4 \\
\hline R96/2569 x R585 (F15) & - & - & 1 & 1 & - & - & - & - & - & - & 2 \\
\hline R98/0814 x R585 (F14) & - & - & 2 & - & - & - & - & - & - & - & 2 \\
\hline R98/4009 x R98/4001 (F38) & 3 & 1 & 1 & 1 & - & 1 & - & - & - & - & 7 \\
\hline R98/6095 x HoCP85/845 (F35) & - & 1 & 1 & - & - & 1 & 1 & - & - & - & 4 \\
\hline RB83/5486 (e) x R575 (F07) & 7 & - & 1 & 4 & 3 & 9 & 6 & - & - & 3 & 33 \\
\hline TC9 x R95/4065 (F36) & - & 1 & - & - & 2 & - & - & - & - & 1 & 4 \\
\hline Total (29) & 14 & 21 & 23 & 15 & 13 & 19 & 11 & 9 & 6 & 8 & 139 \\
\hline
\end{tabular}

\section{DISCUSSION}

Variabilité des caractères quantitatifs: La forte dispersion du poids moyen d'une tige et du nombre de tiges usinables $/ 3 \mathrm{~m}$, d'une part, et la faible dispersion du diamètre des tiges, de la hauteur des tiges et du brix observées chez les génotypes présélectionnées à Ferké est en accord avec les travaux de Tadesse et al (2014) réalisés à Wonji en Éthiopie. La forte dispersion du nombre de tiges usinables/ha a été également rapportée par d'autres auteurs (Chaudhary, 2001) ; Balasundarum and Bhagyalakshmi, 1978; Nair et al, 1980; Sing et Sangwan, 1980). Selon Shivasubramanian and Menon (1973), les valeurs de coefficients de variation phénotypique (CVP) ou génotypique (CVG) sont classées faibles de 0 et $10 \%$, moyennes de 11 à $20 \%$ et fortes 

Stade Ligne en Nouvelle Plantation à Ferké (Nord Côte d'Ivoire)

au-delà de $20 \%$. Les travaux de Singh et al (1994) ont montré que des valeurs élevés de CVP et CVP traduisaient la pertinence des variables phénotypiques observées dans la sélection des variétés et suggéraient une bonne diversité génétique potentielle de celles-ci.

Groupes de variétés déterminés par la CAH : II ressort que chacun des différents groupes de génotypes déterminés par la $\mathrm{CAH}$ à partir des variables quantitatives observées est issu de plusieurs familles dont le nombre varie de 5 (G5) à 16 (G3). Cela montre que chaque groupe est assez diversifié génétiquement. Le nombre de groupes composant chaque famille de clones varie de 1 à 7 suggérant ainsi qu'elle est diversifiée phénotypiquement. La diversité relativement importante observée au sein de chaque groupe de variétés est conforme au fait que les géniteurs des familles étudiées couvrent jusqu'à 7 origines géographiques à travers le monde où la canne à sucre fait l'objet d'hybridations (AfrSud, Réunion, Floride, Hawaï, Maurice, Inde, Argentine). Ces géniteurs sont eux-mêmes des variétés commerciales ou élites (hybrides hétérozygotes) qui sont issues d'hybridations dont les géniteurs couvrent également différentes origines géographiques. Les recombinaisons génétiques à travers ces hybridations permettent d'assurer une diversité variétale assez large pour prendre en compte la diversité des zones de culture de la canne à sucre ciblées, comme rapporté par différents auteurs (Tena et al, 2016 ; Singh et al, 1996; Das et al, 1996; Kumar et Ram, 1996). Elles se manifestent phénotypiquement par nombre de caractères agro-morphologiques, technologiques ou phytosanitaires qui influencent le rendement en canne, la richesse en sucre et le taux d'extraction en sucre. Toutefois, différentes études ont montré que le nombre de groupes obtenus par la $\mathrm{CAH}$ pouvaient varier fortement selon les espèces, le nombre d'accessions et le type ou la quantité de variables observées (Brasileiro et al, 2014 ; Ortiz et al, 2008 ; Gonçalves et al, 2009 ; Barbé et al, 2010 ; Cabral, 2010). Les géniteurs utilisés dans les schémas de sélection ont été obtenus après plusieurs back-cross successifs (sélection récurrente) entre des clones de Saccharum officinarum et ceux d'espèces rustiques de Saccharum dont $S$. spontaneum pour la transmission du caractère richesse saccharine. Dans ce processus, le parent noble utilisé n'est pas nécessairement le même (D'Hont et al, 1998). Toutefois, ces croisements n'ont impliqué qu'un nombre restreint de clones sauvages. Cela suggère que seule une partie de la diversité génétique du genre Saccharum a été utilisée jusque-là dans la sélection de la canne à sucre. Cela conforte le fait que différentes études sur l'évaluation de la diversité génétique par l'utilisation de variables quantitatives et qualitatives, d'une part, et/ou de marqueurs moléculaires, d'autre part, ont révélé une forte similarité génétique entre des variétés différentes avec formation d'un nombre limité de groupes (Da silva et al, 2008 ; Oliveira et al, 2009 ; Duarte Filho et al, 2010 ; Dutra Filho et al, 2011 ; Sindhu et al, 2011 ; Santos et al, 2012 ; Santchurn et al, 2012 ; Perera et al, 2012). C'est pourquoi les schémas de sélection plus récents sont basés sur de nouveaux travaux de nobilisation de visant à introduire de la diversité supplémentaire dans le matériel végétal cultivé. Selon GOUY (2012), la sélection de la canne à sucre est récente car elle commence avec la création des premiers hybrides obtenus à Java au début du siècle dernier. La nobilisation, une méthode de croisements interspécifiques, permit l'obtention de variétés nettement plus performantes et résistantes aux principaux pathogènes de la canne. Depuis, les centres d'hybridation croisent des variétés élites et la sélection se fait à partir du phénotypage des descendances. Utiliser l'information moléculaire pour la sélection d'élites serait une réelle avancée pour le sélectionneur. Bien que de nombreuses études en génétique d'association soient réalisées, aucun marqueur n'est encore utilisé dans les schémas de sélection de la canne à sucre. Les effets des marqueurs ne sont pas estimés de façon assez précise et doivent être validés dans des populations indépendantes. L'association marqueurs-caractères est désormais complétée par de nouvelles approches telles que la sélection génomique et l'utilisation des méthodes écophysiologiques dans la prévision du rendement.

Processus de sélection de la canne à partir des graines : Des milliers de clones différents sont produits chaque année à partir de graines issues du croisement deux à deux de centaines de variétés mâles et femelles, choisies pour leurs caractères complémentaires susceptibles de se combiner. Chaque génération annuelle de clones subit des étapes graduelles de sélection qui s'échelonnent au minimum sur une dizaine d'années. Les épreuves agronomiques, de plus en plus sélectives, permettent d'éliminer les sujets les moins performants. Au terme de la dernière épreuve, un clone élite est retenu pour une éventuelle multiplication commerciale. Les nouvelles variétés élites identifiées à un stade avancé de sélection sont réintroduites dans le programme d'hybridation qui est par ailleurs enrichi par l'importation de matériel végétal d'origine étrangère. Ainsi, de nombreux cycles successifs de sélection, puis d'hybridation, permettent d'assurer une amélioration progressive sur une échelle de plusieurs décennies. La durée du cycle de sélection adoptée en Côte d'Ivoire pour 

Stade Ligne en Nouvelle Plantation à Ferké (Nord Côte d'Ivoire)

la zone agro-écologique de Ferké est de 8 ans au lieu d'au moins 10 ans généralement. Cette durée relativement courte s'explique par la non prise en compte, d'une part, de la phase post-expérimentale consistant en des tests multi-locaux de variétés élites pendant 3 à 4 ans et, d'autre part, des tests pré-commerciaux d'au moins 3 ans sur les 2 principaux types de sols existants (ferralitique, hydromorphe). De plus, les phases de présélection visuelle ont été réduites à 9 mois pour le stade touffe et à 2 ans pour le stade ligne. La phase expérimentale avec un dispositif statistique généralement en blocs complets randomisés s'étend sur 5 ans dont 2 ans pour la 1ère sélection et 3 ans pour la $2^{\text {ème }}$ sélection. La phase post-expérimentale multi-locale a, quant à elle, été supprimée car les deux complexes sucriers de Ferké sont situés dans la même zone agro-écologique. Le principal critère de sélection est le rendement en saccharose/ha qui constitue la variable la plus directement corrélée au profit. Le second critère porte sur la tolérance ou la résistance aux maladies et aux ravageurs endémiques. Le troisième critère, dans l'ordre de priorité, est la tenue en repousse afin de mieux amortir le coût d'investissement d'une nouvelle plantation. Outre ces critères généraux de sélection, certains pays considèrent des critères plus spécifiques basés sur les pratiques culturales particulières (récolte mécanisée en vert, récolte en fin de campagne sucrière,), l'usinage de la canne et la production d'énergie (teneur élevée en fibre) ou l'adaptation à des conditions agro-écologiques typées. Le succès des industries sucrières situées dans les pays qui ne sont pas obtenteurs de variétés dépend de l'introduction de génotypes performants d'origine étrangère. Les chances d'adaptation d'une variété donnée à l'étranger sont d'abord liées aux similitudes agropédoclimatiques existant entre le pays obtenteur et le pays utilisateur. Plus les similitudes ne sont proches, meilleures sont les chances d'adaptation. II importe de souligner qu'il existe un bon esprit de collaboration entre les stations d'hybridation regroupées au sein de l'ISSCT où les échanges réciproques de cultivars ou de génotypes élites sont très fréquents. De plus, les ressources génétiques disponibles dans les collections

\section{CONCLUSION}

L'étude a montré que les variables quantitatives permettant de mieux expliquer la diversité des clones présélectionnées au stade ligne de $3 \mathrm{~m}$ comprenaient, dans l'ordre décroissant, le poids moyen d'une tige, le diamètre moyen des tiges, la longueur moyenne des tiges, le nombre de tiges usinables $/ 3 \mathrm{~m}$ et le brix réfractométrique. Ces variables ont fortement contribué à variétales de canne sous l'égide de l'ISSCT sont mises à contribution au profit de toutes les stations d'hybridation. Ces traditions d'échanges ouverts favorisent l'exploitation de la diversité génétique la plus large. Les risques phytosanitaires liés à la circulation des boutures sont contrôlés par le passage obligatoire du matériel végétal dans des quarantaines sécurisées.

Progrès génétique du rendement: Les travaux de Simmonds (1979) sur la succession des clones plantés à Barbade entre 1940 et 1975, ont permis de comparer le rendement et canne et la richesse saccharine des nouvelles variétés par rapport aux précédentes durant les années de transition où coexistaient deux générations successives. Cela lui a permis de distinguer, dans les gains de productivité obtenus, l'effet attribuable à l'amélioration génétique des effets dus aux fluctuations climatiques ou à l'évolution des pratiques agricoles. De cette façon, il a estimé sur la durée de l'étude (35 ans) le progrès génétique annuel à $0.64 \%$. Sur une durée analogue, Hogarth (1971), cité par Heinz (1987), a évalué à $1 \%$ le progrès génétique moyen annuel en culture de canne dans le Queensland. À Hawaï, les gains moyens annuels dus à l'amélioration variétale depuis 1955 ont été du même ordre (Heinz, 1987). Ponctuellement, quelques variétés exceptionnelles ont montré des gains très élevés par rapports aux variétés témoins. Ça été le cas en Australie dans les années 1970 avec la variété Q90 qui a supplanté le rendement en sucre de la variété Pindar au Nord du pays de $31 \%$ (Berding et Skinner, 1987). Un gain comparable a été réalisé à La Réunion dans les années 1980 avec la variété R570 (Hellmann, 1984). Plus récemment, le Queensland a connu une progression spectaculaire de rendement d'environ $20 \%$ depuis la fin des années 1980, principalement grâce à la diffusion des variétés Q124, CP51-21, Q141 et Q146 (Cox et Hansen, 1995). La supériorité d'une nouvelle variété par rapport aux précédentes se mesure surtout par la longévité de son adoption en exploitation agricole. Le caractère qui détermine son succès réside surtout dans sa tenue en repousse avec des rendements élevés en canne et en sucre.

la discrimination de 10 groupes phénotypiques de clones. Cela suggère une assez bonne diversité génétique parmi les 139 clones de canne à sucre présélectionnés. Les 4 groupes les mieux représentés (G3, G2, G6 et G4 avec $56 \%$ de l'effectif total) comprenaient chacun 15 à 23 individus, tandis que les moins représentés (G7, G8, G9 et $\mathrm{G} 10$ avec $24.5 \%$ du total) 6 à 11 individus chacun. Les 
variables qualitatives les plus pertinentes dans la discrimination des groupes concernaient la densité du tallage et l'intensité de la floraison. Les clones du groupe G1 étaient associés à un tallage très fort voire excellent et

\section{RÉFÉRENCES CITÉES}

Amalraj VA and Balasundaram N, 2009. Status of sugarcane genetic resources in India, PGR Newsletter $148: 26-31$.

Balasundaram N, Bhagyalkshmi KV, 1978. Variability, heritability and association among yield and yield components in sugarcane. Indian J. Agric. Sci. 48: 291-295.

Barbé TD, Gonçalves LSA, Rodrigues R, 2010. Association between advanced generations and genealogy in inbred lines of snap bean by the Ward-modified location model. Euphytica 173: 337-343.

Benzécri JP, 1970. Leçon sur l'analyse statistique des données multidimensionnelles. Faculté des sciences de paris.

Berding N, Skinner JC, 1987. Traditional breeding methods. In : Proc. Coopersucar Int. Sugarcane Breeding Workshop, Piracicaba- SP, Brasil : 269-320.

Brasileiro BP, Marinho CD, Costa PMA, Moreira EFA, Peternelli LA, Barbosa MHP, 2014. Genetic diversity in sugarcane varieties in Brazil based on the Ward-Modified Location Model clustering strategy. Genet. Mol. Res. 13 (1) : 1650-1660.

Cabral PDS, Soares TCB, Gonçalves LSA, Amaral AT, 2010. Quantification of the diversity among common bean accessions using Ward-MLM strategy. Pesa. Agropec. Brasil 45: 1124-1132.

Chaudhary RR, 2001. Genetic variability and heritability in sugarcane. Nepal Agric. Res. J. Vol $4 \& 5$ : xxx$\mathrm{xxx}$.

Cox MC, Hansen PB, 1995. Productivity trends in southern and central regions, and the impact of new varieties. Proc. Austr. Soc. Of Surgarcane Technol. 17: 1-7.

Da silva CM, Mangolin CA, Mott AS, Machado MPS, 2008. Genetic diversity associated with in vitro and conventional bud propagation of Saccharum varieties using RAPD analysis. Plant Breed. 127: 160-165.

Das Pk, Nayak BC, Nayak N, Parida AK, 1996. Genetic variability in biometrical trials of early maturing genotypes of sugarcane. Indian Sugar 46: 723726. une forte intensité de floraison. Par contre, ceux des groupes G5, G7, G8 et G10 tendaient à présenter un tallage moyen à faible et une très faible intensité de floraison.

Duarte Filho L, Silva P, Santos J, Barbosa G, 2010. Genetic similarity among genotypes of sugarcane estimated by SSR and coefficient of parentage. Sugar Tech. 12: 145-149.

Gashaw ET, Mekbib F, Ayana A, 2016. Genetic diversity among sugarcane genotypes based on qualitative traits. Advances in Agriculture Article ID 8909506, 8p.

Gonçalves Ls, Rodrigues R, do Amaral Jr AT, Karasawa $M, 2009$. Heirloon tomato gene bank : assessing genetic divergence based on morphological, agronomic and molecular data using a Wardmodidied location model. Gent. Mol. Res. 8: 364-374.

Hellmann M, 1987. Contrôle informatique des champs. In : Rapport annuel 1984 du CERF : 46-51.

Heinz DJ, 1987. Introduction. In : Heinz DJ. Sugarcane improvement through breeding. Elsevier Press. Amsterdam : 1-5.

Hoarau JY, Payet J, Hellmann M, 2002. Un siècle d'amélioration génétique de la canne à sucre à travers le monde. Un aperçu de la progression des rendements agricoles. Communication personnelle, $10 \mathrm{p}$.

Hogarth D, 1971. Quantitative inheritance studies in sugarcane: II. Correlations and predicted responses to selection. Austral. J Agric. Res. 22: 103-109.

Kouamé KD, Péné CB, Zouzou M, 2010. Évaluation de la résistance variétale de la canne à sucre au foreur de tiges tropical africain (Eldana saccharina Walker) en Côte d'lvoire. J. of Applied Biosci. 26: 1614-1622.

Kumar S, Ram B, 1996. Genetic variability, heritability and correlations in hybrid $x$ species progenies of sugarcane grown under saline conditions. Sugarc. 2: 17-19.

Nair NV, Somarajan KG, Balasundaram N, 1980. Genetic variability, heritability and genetic advance in Saccharun officinarum. Int. Sugarc. J. XXXII : 275-276.

Oliveira KM, Pinto LR, Marconi TG, Mollinari M, 2009. Characterization of new polymorphic functional markers for sugarcane. Genome 52: 191-209. 
Ortiz R, Sevilla R, Alvarado G, Crossa J, 2008. Numerical classification of related Peruvian highland maize races using internal ear traits. Genet. Resour. Crop. Ev. 55: 1055-1064.

Péné CB, Déa GB, 2000. Amélioration de la canne à sucre en Côte d'Ivoire. In : 1er atelier régional sur l'amélioration variétale de la canne à sucre en Afrique de l'Ouest et Centrale tenu en Octobre 1999 à Yamoussoukro. Montpellier, CIRAD : 98 $-102$.

Péné CB, Kouamé DK, Dove H, Boua MB, 2016. Incidence des infestations du foreur de tiges Eldana saccharina (Lepidoptera, Pyralidea) en culture irriguée de canne à sucre selon la variété et la période de récolte en Côte d'lvoire. J. of Applied Biosci. 102: 9687 - 9698.

Péné CB, Tuo K, 2007. Early and late-season screening of sugarcane varieties in north Ivory Coast, Annual report, 77 pages.

Perera MF, Arias ME, Costilla D, luque AC, 2012. Genetic diversity assessment and genotype identification in sugarcane based on DNA markers and morphologial traits. Euphytica 185: 491-510.

Santchurn D, Ramdoyal K, Badaloo MGH, Labuschagne $M, 2012$. From sugar industry to cane industry: investigations on multivariate data analysis technique in the identification of different high biomass sugarcane varieties. Euphytica 185: 543-558.

Santos JM, Durte Filho LSC, Soriano ML, Silva PP, 2012. Genetic diversity of the main progenitors of sugarcane from the RIDESA germplasm bank using SSR markers. Ind. Crop. Prod. 40: 145150.

Shivasubramanian S, Menon M, 1973. Heterosis and inbreeding depression in rice. Madra Agric. J. 60: 1139

Simmonds NW, 1979. The impact of plant breeding on sugarcane yields in Barbados. Tropical Agric. Trinidad 56 (4): 289-300.

Sindhu R, Govindaraj P, Balamurugan A, Appupnu C, 2011. Genetic diversity in sugarcane hybrids (Saccharum spp complex) grown in tropical India based on STMS markers. J. Plant Biochem. Biol. 20: 118-124.

Singh AP, Chatterjee A, Nema GK, 1996. Genetic variability in sugarcane (Saccharum spp. complex). Crop Res. (Hisar) 12: 162-164.
Singh R, Sangwan RS, 1980. Studies on genetic variability for stalk characters in sugarcane. Indian Sugar 30: 409-412.

Singh RK, Singh DN, Singh SK, Singh HN, 1994. Genetic variability and correlation studies in foreign commercial hybrids of sugarcane. Agric. Sci. Dig., Karnal: 103-107.

Sivaneanesan A, Waller JM, 1986. Sugarcane diseases, CMI Phytopathology Paper 29, CAB International, Wallingford, UK.

Tadesse F, Negi T, Getaneh A, Dilnesaw Z, Ayele N, Teferi Y, 2014. Genetic variability and heritability of ten exotic sugar cane genotypes at Wonji Sugar Estate of Ethiopia. Global Advanced Research J. Physic. Appl. Sci. Vol 3(4):

Tena E, Mekbib F, Ayana A, 2016. Genetic diversity of quantitative traits of sugarcane genotypes in Ethiopia. Americ. J. Plant Sci. 7: 1498-1520.

Thomas LT, 2008. Improvement of bioenergy Crops, Springer Science, New York. NY, USA.

Volle M, 1997. L'analyse des données. In : Économie et statistique. Institut National de la Statistique et des Études économiques 96 (1): 3-23.

Zadi M., Turquin L., Péné CB, 2017. Évaluations agronomiques et technologiques des clones de canne à sucre (Saccharum officinarum L.) présélectionnés au stade ligne à Ferkessédougou, Nord Côte d'lvoire. J. Appl. Biosci. 115 : 11502 - 11512. 